\title{
An Inclusive Comparison in LAN Environment between Conventional and Hybrid Methods for Spectral Amplitude Coding Optical CDMA Systems
}

\author{
Hassan Yousif Ahmed \\ Electrical Engineering Department \\ College of Engineering at Wadi Aldawaser, PSAU \\ Wadi Aldawasir, KSA
}

\author{
Nisar K.S \\ Mathematics Department \\ College of Art and Science, PSAU \\ Wadi Aldawasir, KSA
}

\author{
Z. M Gharsseldien \\ Mathematics Department \\ College of Art and Science, PSAU \\ Wadi Aldawasir, KSA
}

\author{
S. A. Aljunid \\ School of Computer and Communication Engineering \\ Universiti Malaysia Perlis \\ Kangar, Malaysia
}

\begin{abstract}
In this paper, performance analysis of conventional spectral amplitude coding (CSAC) with hybrid (HSAC) for OCDMA system is investigated in local area network (LAN) environment. The CSAC is built based on arithmetic sequence with simple algebraic ways. The HSAC technique is used in which spectral amplitude coding (SAC) combined with wavelength division multiplexing (WDM) to effectively reduce multiple access interference (MAI) and mitigate the influence of phase induced intensity noise (PIIN) arising in photodetecting process. The main idea is to construct the code sequences in SAC domain then repeat it diagonally in the wavelength domain as groups which maintains the same cardinality of a given code weight. Results show that HSAC outperforms CSAC when the number of active users is high due to its better correlation properties. It has been shown that the HSAC can suppress intensity noise effectively and improve the bandwidth utilization significantly up to $4.2 \mathrm{~nm}$.
\end{abstract}

Keywords-Conventional SAC (CSAC); Hybrid SAC (HSAC); WDM; OCDMA; MAI

\section{INTRODUCTION}

The demand for networks with higher capabilities at lower cost is increasing daily. This demand is fueled by many different factors. The tremendous growth of the internet has brought huge amount of users consuming large amount of bandwidth since data transfers involving video and image. To fulfill the demands for bandwidth and to deploy new services, new technology must deployed and fiber optic is such one key technology. There is not much different between multiple access and multiplexing techniques, in simple word, multiple access is a technique that allows communication media to be shared between different users while multiplexing is a combination of signals into single transmission signal [1]. Wavelength division multiplexing (WDM) has been considered as an ideal solution to extend the capacity of optical networks without drastically changing the fiber infrastructure. Various architectures that incorporate WDM into access networks have been proposed by both academia and industry [1-2]. Optical code division multiple access (OCDMA) has been considered lately as an efficient scheme for optical communication networks [3]. Among all OCDMA techniques, spectral amplitude coding (SAC) systems have been considered since multiple access interference (MAI) can be completely eliminated by spectral coding [4-6]. However, as long as the number of users increases, phase induced intensity noise (PIIN) arises during balance photodetecting process which results in higher bit error probability. This eventually limits the network capacity. Several schemes have been introduced to be combined with OCDMA technique to improve the MAI cancellation and support more users in fiber optical networks [7-13]. Among all of them temporal/spatial OCDMA networks to improve autocorrelation sidelobes and cross-correlation have been proposed in [7]. Applying an optical pulse to represent one chip in the wavelength and time domain is one way of the MAI improvement schemes [8-10]. Some proposals [11-13] have employed differential detection to reduce the MAI. However, these systems are suffered from different problems one way or another to remove all the MAI at the receiver, resulting in severe interference, which limits the number of active users in the network. In SAC system, fiber Bragg grating (FBG) can be used as main part of encoder-decoder structure of each user. When the number of active users becomes large the sizes of FBG will become impractical. One way to relax impracticality of FBGs is to use two dimensional coding schemes but at the cost of additional fiber ribbons and star couplers are required [14]. In this paper, firstly a conventional SAC (CSAC) code family is built by using simple algebraic ways. Secondly a hybrid SAC scheme is modeled by combining WDM and SAC which maintains MAI cancellation property and PIIN alleviation in OCDMA network. The CSAC code sequences is characterized by the parameters $L, N, W, \lambda_{\mathrm{c}}$, where $L$ is the code length, $N$ is the number of users, $W$ is the code weight (number of marks) and $\lambda \mathrm{c}$ is the cross correlation $\left(\lambda_{\mathrm{c}}=1\right)$. The CSAC scheme is built with fixed in-phase cross correlation aiming to completely remove the MAI by differential detection at the receiver side. 
Block $_{\mathrm{W}} \quad$ Block $_{1} \quad$ Block $_{2} \quad$ Block $_{3} \quad----$--.-. $\quad$ Block $_{(\mathrm{w}-1)}$

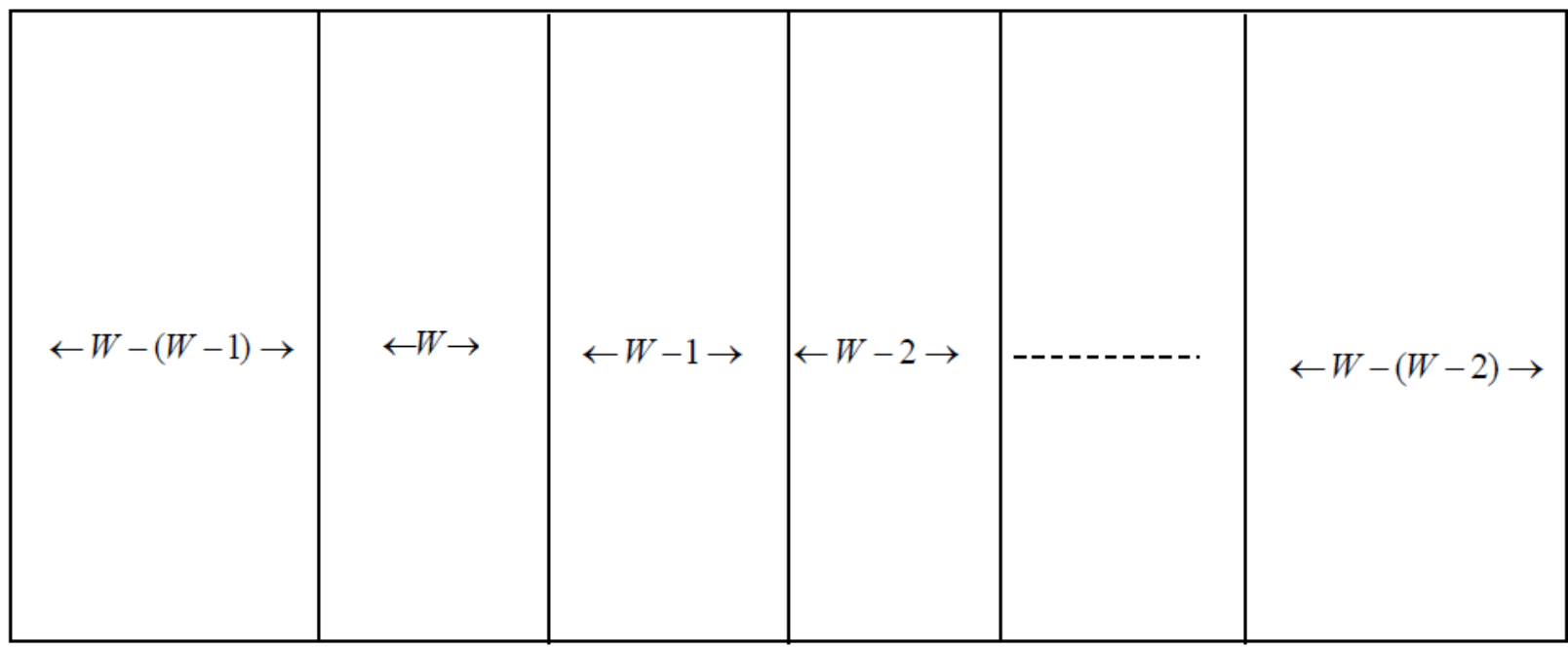

Fig. 1. Mapping elements in AS to blocks in CSAC matrix

The PIIN with the power square at photodiodes (PDs) is noticeably suppressed due to the space for spreading the received power which makes the PDs get less power for the PIIN, while maintaining the same signal power in the receiver [4], [15]. The rest of the paper is organized as follows. Section 2 shows steps of construction of CSAC code family. The HSAC model is described in Section 3. The HSAC encoder/decoder structure is explained in Section 4. In Section 5 , the performance analysis of the CSAC and HSAC in the OCDMA network is elaborated. Theoretical analysis and numerical results are presented in Section 6. Finally, the conclusions are drawn in Section 7.

\section{CODE CONSTRUCTION AND PROPERTIES}

\section{A. Definitions}

Let $A S=(W, W-1, W-2, W-3, W-4, \ldots, 1)$ denotes an arithmetic sequence. The sum of $W$ terms of the arithmetic sequence (AS) can be calculated by.

$$
S_{w}=\frac{W}{2}(W+1)
$$

The value of $S_{\mathrm{w}}$ represents the number of columns of conventional SAC (CSAC). Fig. 1 illustrates the mapping process of AS to CS, where each element in AS will be mapped to corresponding block in CSAC. The length of each block can be calculated by the formula.

$$
\mathrm{Lg}=W-g+1
$$

where $W$ is the code weight and $g=1,2,3, \ldots, N$

\section{B. Construction steps of CSAC code family}

Step1: Form the arithmetic sequence (AS) as follows.

$$
A S=(W, W-1, W-2, W-3, W-4, \ldots, 1)
$$

Step 2: Calculate value(s) of $m$, such that:

$$
M_{1} \leq m \leq M_{2}
$$

where,

$$
\begin{gathered}
M_{1}=2+(r-1)\left(W-\frac{r-2}{2}\right) \\
M_{2}=1+r\left(W-\frac{r-1}{2}\right)
\end{gathered}
$$

Notice that, if the value of $M_{1}$ exceeds the value of $L$ then $\mathrm{m}$ carries the value 1 (i.e., if $M_{1}>L$ then $m, M_{1}, M_{2}$ assign the value 1 ).

Step 3: Calculate the code length

$$
L=\frac{(W \times N)}{2}
$$

Step 4: Calculate the position of " $1 \mathrm{~s}$ " in the first row of each block using

$$
p=(r, m)
$$

Step 5: Calculate the positions of cross-correlated " $1 \mathrm{~s}$ " in each block using

$$
q=\left(r+m-M_{1}+1, m\right)
$$

Step 6: Fill each row with $\frac{W(N-2)}{W-1} " 0 s "$, where $N$ is the number of users.

\section{Code Examples}

To clarify the steps mentioned in Section 2.2, we consider the following two cases.

Case 1: for $W=3, N=4$

Step1: Form the arithmetic sequence, so the block of CSAC is $r=1,2,3$.

Step 2: Calculate $M_{1}$ and $M_{2}$ using Eq. (4) and Eq. (5). For $r=1$,

Identify applicable sponsor/s here. If no sponsors, delete this text box (sponsors). 
$M_{1}=2+(1-1)\left(3-\frac{1-2}{2}\right)=2$

$M_{2}=1+1\left(3-\frac{1-1}{2}\right)=4$

hence $\mathrm{m}=2,3,4$ using (2).

For $r=2$,

$M_{1}=2+(2-1)\left(3-\frac{2-2}{2}\right)=5$

$M_{2}=1+2\left(3-\frac{2-1}{2}\right)=6$

hence $m=5,6$ using (2).

For $r=3$,

$M_{1}=2+(3-1)\left(3-\frac{3-2}{2}\right)=7$

$M_{2}=1+3\left(3-\frac{3-1}{2}\right)=7$

hence $\mathrm{m}, M_{1}, M_{2}=1$ as $M_{1}>L$.

Step 3: Calculate the code length using (6).

$L=\frac{(3 \times 4)}{2}=6$

Step 4: Calculate $p$ using (7)

For $r=1$ and $m=2, p=(1,2)$

For $r=1$ and $m=3, p=(1,3)$

For $r=1$ and $m=4, p=(1,4)$

For $r=2$ and $m=5, p=(2,5)$

For $r=2$ and $m=6, p=(2,6)$

For $r=3$ and $m=1, p=(3,1)$

Step 5: Calculate q using (8)

For $r=1, M_{1}=2$ and $m=2,3,4$

$q=(1+2-2+1,2)=(2,2)$

$q=(1+3-2+1,3)=(3,3)$

$q=(1+4-2+1,4)=(4,4)$

For $r=2, M_{1}=5$ and $m=5,6$.

$q=(2+5-5+1,5)=(3,5)$

$q=(2+6-5+1,6)=(4,6)$

For $r=3, M_{1}=1$ and $\mathrm{m}=1\left(\right.$ as $\left.M_{1}>\mathrm{L}\right)$

$q=(3+1-1+1,1)=(4,1)$

Step6: Pad each raw with a $\frac{W(N-2)}{2} " 0 s^{\prime \prime}$

$=\frac{3(4-2)}{2}=\frac{3(2)}{2}=3$ “0s".

Using the above steps of construction in Section 2.2, we have listed some code sequences in Eq. (10). In this equation, the coordinates of $p$ obtained in step 3 are $(1,2),(1,3),(1,4)$, $(2,5),(2,6)$, and $(3,1)$ while the coordinates of $q$ obtained in step 4 are $(2,2),(3,3),(4,4),(3,5),(4,6)$, and $(4,1)$. It should be pointed out that, all these coordinates represent the positions of " $1 \mathrm{~s}$ " whereas the positions of " $0 \mathrm{~s}$ " calculated by using step 6 . Therefore, using step 4, step 5 and step 6, the code patterns can be generated

$$
C S A C=\left[\begin{array}{llllll}
0 & 1 & 1 & 1 & 0 & 0 \\
0 & 1 & 0 & 0 & 1 & 1 \\
1 & 0 & 1 & 0 & 1 & 0 \\
1 & 0 & 0 & 1 & 0 & 1
\end{array}\right]
$$

Case 2: for $W=4, N=5$

Step1: Form the arithmetic sequence

$A S=(4,4-1,4-2,4-3)=(4,3,2,1)$

, so the block of CSAC is $r=1,2,3,4$.

Step 2: Calculate $M_{1}$ and $M_{2}$ using Eq. (4) and Eq. (5).

For $r=1$,

$M_{1}=2+(1-1)\left(4-\frac{1-2}{2}\right)=2$

$M_{2}=1+1\left(4-\frac{1-1}{2}\right)=5$

hence $\mathrm{m}=2,3,4,5$ using (2).

For $\mathrm{r}=2$,

$M_{1}=2+(2-1)\left(4-\frac{2-2}{2}\right)=6$

$M_{2}=1+2\left(4-\frac{2-1}{2}\right)=8$

hence $m=6,7,8$ using (2).

For $\mathrm{r}=3$,

$M_{1}=2+(3-1)\left(4-\frac{3-2}{2}\right)=9$

$M_{2}=1+3\left(4-\frac{3-1}{2}\right)=10$

hence $\mathrm{m}=9,10$ using (2).

For $r=4$,

$$
\begin{aligned}
& M_{1}=2+(4-1)\left(4-\frac{4-2}{2}\right)=11 \\
& M_{2}=1+4\left(4-\frac{4-1}{2}\right)=11
\end{aligned}
$$


Step 3: Calculate the code length using (6).

$$
L=\frac{(4 \times 5)}{2}=10
$$

hence $m=M_{1}=M_{2}=1$ as $M_{1}>L$.

Step4: Calculate p using (7)

For $r=1$ and $m=2, p=(1,2)$

For $r=1$ and $m=3, p=(1,3)$

For $r=1$ and $m=4, p=(1,4)$

For $r=1$ and $m=5, p=(1,5)$

For $r=2$ and $m=6, p=(2,6)$

For $r=2$ and $m=7, p=(2,7)$

For $r=2$ and $\mathrm{m}=8, p=(2,8)$

For $r=3$ and $\mathrm{m}=1, p=(3,9)$

For $r=3$ and $\mathrm{m}=1, p=(3,10)$

For $r=4$ and $m=1, p=(4,1)$

Step 5: Calculate q using (8)

For $r=1, M_{1}=2$ and $m=2,3,4,5$.

$$
\begin{aligned}
& q=(1+2-2+1,2)=(2,2) \\
& q=(1+3-2+1,3)=(3,3) \\
& q=(1+4-2+1,4)=(4,4) \\
& q=(1+5-2+1,5)=(5,5)
\end{aligned}
$$

For $r=2, M_{1}=6$ and $m=6,7,8$.

$$
\begin{aligned}
& q=(2+6-6+1,6)=(3,6) \\
& q=(2+7-6+1,7)=(4,7) \\
& q=(2+8-6+1,8)=(5,8)
\end{aligned}
$$

For $r=3, M_{1}=9$ and $\mathrm{m}=9,10$.

$$
\begin{aligned}
& q=(3+9-9+1,9)=(4,9) \\
& q=(3+10-9+1,10)=(5,10)
\end{aligned}
$$

For $r=4, M_{1}=1$ and $\mathrm{m}=1\left(\operatorname{as} M_{1}>L\right)$

$q=(4+1-1+1,1)=(5,1)$

Step6: Pad each raw with a

$\frac{W(N-2)}{2} " 0 s^{\prime \prime}=\frac{4(5-2)}{2}=\frac{4(3)}{2}=6 " 0 \mathrm{~s} "$

Using the above steps of construction in Section 2.2, we have listed some code sequences in Eq. (11). In this equation, the coordinates of $p$ obtained in step 3 are $(1.2),(1,3),(1,4)$ $(1,5)(2,6)(2,7)(2,8)(3,9)(3,10)$ and $(4,1)$ while the coordinates of $q$ obtained in step 4 are $(2,2),(3,3),(4,4),(5,5)$, $(3,6),(4,7),(5,8),(4,9),(5,10)$, and $(5,1)$. It should be pointed out that, all these coordinates represent the positions of " $1 \mathrm{~s}$ " whereas the positions of " $0 \mathrm{~s}$ " calculated by using step 6 .

Therefore, using step 4, step 5 and step 6 , the code patterns can be generated as follows.

$$
C S A C=\left[\begin{array}{llllllllll}
0 & 1 & 1 & 1 & 1 & 0 & 0 & 0 & 0 & 0 \\
0 & 1 & 0 & 0 & 0 & 1 & 1 & 1 & 0 & 0 \\
0 & 0 & 1 & 0 & 0 & 1 & 0 & 0 & 1 & 1 \\
1 & 0 & 0 & 1 & 0 & 0 & 1 & 0 & 1 & 0 \\
1 & 0 & 0 & 0 & 1 & 0 & 0 & 1 & 0 & 1
\end{array}\right]
$$

\section{MODEL DESCRIPTION OF HSAC SYSTEM}

$$
\left[\begin{array}{ccccc}
(C S A C)_{1} & 0 & 0 & \cdots & 0 \\
0 & (C S A C)_{2} & 0 & \cdots & 0 \\
0 & 0 & \ddots & \ddots & \vdots \\
\vdots & \vdots & \ddots & \ddots & 0 \\
0 & 0 & \cdots & 0 & (C S A C)_{N}
\end{array}\right]
$$

Fig. 2. Matrix representation of HSAC code

An HSAC is a system where the whole code constructed in SAC domain (CSAC) then repeated in a diagonal fashion in the wavelength domain as groups. Each group maintains the same cardinality of a given code's weight of SAC code as shown in Fig. 2. In this HSAC system, the code words are divided in to $g$ groups, where $g=1,2,3, \ldots$ Each user indexed as user \# $(z, t)$ and assigned a code sequence $C_{z, t}, z=1,2,3 \ldots \ldots, g$ and $t$ $=1,2,3 \ldots ., N$. The code length $L$ can be calculated using the formula (11).

$$
L=g \frac{(W \times N)}{2}
$$

In Eq. (12) and Eq. (13) $p$ and $q$ represent the positions of " $1 \mathrm{~s}$ " in the first row of each block and the positions of crosscorrelated " $1 \mathrm{~s}$ " in each block respectively. $p=(r+(g-1) N, m+(g-1) L)$ 
$q=\left(r+m-M_{1}+(g-1) N+1, m+(g-1) L\right)$

To clarify HSAC system let us consider the following two cases.

Case 1: for $W=3, N=8$ and $g=2$

Using the steps $1,2,5$ in Section II- $B$ and equations (13) and (14) in Section III to calculate the position of " $1 \mathrm{~s}$ " in the first row of each block and the positions of cross-correlated "1s" in each block respectively (see Fig.1), where Table.I can be easily generated. Therefore, the points coordinates obtained for $p$ are $(1,2),(1,3),(1,4),(2,5),(2,6),(3,1)$ using Eq. (7) and $(5,8),(5,9),(5,10),(6,11),(6,12),(7,7)$ using Eq. (13). While for $q$ are $(2,2),(3,3),(4,4),(3,5),(4,6),(4,1)$ using $(8)$ and $(6,8),(7,9),(8,10),(7,11),(8,12),(8,7)$ using Eq. $(14)$.

TABLE I. HSAC CODE WORDS FOR $\mathrm{G}=2$ AND $\mathrm{N}=8$

\begin{tabular}{|c|l|l|l|l|l|l|l|l|l|l|l|l|l|}
\hline$z$ & $t$ & \multicolumn{10}{|c|}{ HSAC code sequences $\mathrm{C}_{\mathrm{z}, \mathrm{t}}$} \\
\hline 1 & 1 & 0 & 1 & 1 & 1 & 0 & 0 & 0 & 0 & 0 & 0 & 0 & 0 \\
\hline 1 & 2 & 0 & 1 & 0 & 0 & 1 & 1 & 0 & 0 & 0 & 0 & 0 & 0 \\
\hline 1 & 3 & 1 & 0 & 1 & 0 & 1 & 0 & 0 & 0 & 0 & 0 & 0 & 0 \\
\hline 1 & 4 & 1 & 0 & 0 & 1 & 0 & 1 & 0 & 0 & 0 & 0 & 0 & 0 \\
\hline 2 & 5 & 0 & 0 & 0 & 0 & 0 & 0 & 0 & 1 & 1 & 1 & 0 & 0 \\
\hline 2 & 6 & 0 & 0 & 0 & 0 & 0 & 0 & 0 & 1 & 0 & 0 & 1 & 1 \\
\hline 2 & 7 & 0 & 0 & 0 & 0 & 0 & 0 & 1 & 0 & 1 & 0 & 1 & 0 \\
\hline 2 & 8 & 0 & 0 & 0 & 0 & 0 & 0 & 1 & 0 & 0 & 1 & 0 & 1 \\
\hline
\end{tabular}

Case 2: for $W=4, N=10$ and $g=2$

Using the steps $1,3,6$ in Section 2.1 and equations (12), (13) in section 3 to calculate the position of " $1 \mathrm{~s}$ " in the first row of each block and the positions of cross-correlated " $1 \mathrm{~s}$ " in each block respectively (see Fig.1), where Table. II can be easily generated. Therefore, the points coordinates obtained for $p$ are $(1.2),(1,3),(1,4)(1,5)(2,6)(2,7)(2,8)(3,9)(3,10)$ $(4,1)$ using Eq. (7) and $(5,8),(5,9),(5,10),(6,11),(6,12),(7,7)$ using Eq. (12). While for $q$ are $(2,2),(3,3),(4,4),(5,5),(3,6)$, $(4,7),(5,8),(4,9),(5,10),(5,1)$ using $(8)$ and $(6,8),(7,9),(8,10)$, $(7,11),(8,12)$, and $(8,7)$ using Eq. (14).

\section{CONFIGURATION OF TRANSMITTER AND RECEIVER SECTIONS}

Table II shows an example of the HSAC code words of two groups ( 8 users) are obtained by applying the steps of code construction mentioned in Section II-B.
TABLE II. CODE WORDS FOR $\mathrm{G}=2$ AND $\mathrm{N}=10$

\begin{tabular}{|c|c|c|c|c|c|c|c|c|c|c|c|c|c|c|c|c|c|c|c|c|c|}
\hline & $t$ & \multicolumn{20}{|c|}{ HSAC code sequences $\mathrm{Cz}, \mathrm{t}$} \\
\hline 1 & 1 & 0 & 1 & 1 & 1 & 1 & 0 & 0 & 0 & 0 & 0 & 0 & 0 & 0 & 0 & 0 & 0 & 0 & 0 & 0 & 0 \\
\hline 1 & 2 & O & 1 & 0 & 0 & 0 & 1 & 1 & 1 & 0 & 0 & 0 & 0 & 0 & 0 & 0 & 0 & 0 & 0 & 0 & 0 \\
\hline 1 & 3 & 0 & 0 & 1 & 0 & 0 & 1 & 0 & 0 & 1 & 1 & 0 & 0 & 0 & 0 & 0 & 0 & 0 & 0 & 0 & 0 \\
\hline 1 & 4 & 1 & 0 & 0 & 1 & 0 & 0 & 1 & 0 & 1 & 0 & 0 & 0 & 0 & 0 & 0 & 0 & 0 & 0 & 0 & 0 \\
\hline 1 & 5 & 1 & 0 & 0 & 0 & 1 & 0 & 0 & 1 & 0 & 1 & 0 & 0 & 0 & 0 & 0 & 0 & 0 & 0 & 0 & 0 \\
\hline 2 & 6 & 0 & 0 & 0 & 0 & 0 & 0 & 0 & 0 & 0 & 0 & 0 & 1 & 1 & 1 & 1 & 0 & 0 & 0 & 0 & 0 \\
\hline 2 & 7 & 0 & 0 & 0 & 0 & 0 & 0 & 0 & 0 & 0 & 0 & 0 & 1 & 0 & 0 & 0 & 1 & 1 & 1 & 0 & 0 \\
\hline 2 & 8 & 0 & 0 & 0 & 0 & 0 & 0 & 0 & 0 & 0 & 0 & 0 & 0 & 1 & 0 & 0 & 1 & 0 & 0 & 1 & 1 \\
\hline 2 & 9 & 0 & 0 & 0 & 0 & 0 & 0 & 0 & 0 & 0 & 0 & 1 & 0 & 0 & 1 & 0 & 0 & 1 & 0 & 1 & 0 \\
\hline 2 & $\begin{array}{l}1 \\
0\end{array}$ & 0 & 0 & 0 & 0 & 0 & 0 & 0 & 0 & 0 & U & 1 & U & 0 & 0 & 1 & 0 & 0 & 1 & 0 & 1 \\
\hline
\end{tabular}

Table III represents the corresponding amplitude spectra of the code sequences shown in Table I, where the violet bellshaped curves represent " $1 \mathrm{~s}$ " chips and the blank spaces represent " $0 \mathrm{~s}$ " chips. The in phase cross correlation between codes words within the same group (from Table III, $\mathrm{C}_{(1,1)}$, $\left.\mathrm{C}_{(1,2)}, \mathrm{C}_{(1,3)}, \mathrm{C}_{(1,4)}\right)$ is one $(\lambda=1)$, while the codes from different groups ( from Table III, $\left.\mathrm{C}_{(2,5)}, \mathrm{C}_{(2,6)}, \mathrm{C}_{(2,7)}, \mathrm{C}_{(2,8)}\right)$ is zero $(\lambda=$ $0)$.

From Table III we can observe that the CSAC code has six spectral bins while HSAC code has twelve spectral bins. The bin bandwidth for CSAC code is set to $0.4 \mathrm{~nm}$, which results in a $2.4 \mathrm{~nm}$ total bandwidth $(\mathbf{1 5 4 7 . 2} \mathrm{nm}$ to $1549.2 \mathrm{~nm})$. In the meantime, the bin bandwidth of HSAC codes were set to 0.4 $\mathrm{nm}$ resulting in $4.8 \mathrm{~nm}$ total bandwidth $(\mathbf{1 5 4 9 . 6} \mathbf{~ n m}$ to 1551.6 nm).

The transmitter/receiver structure based on the HSAC code sequence for $W=3$ is shown Fig. 3. The principle idea behind the HSAC detection technique is that only intended signal spectrum and overlapping spectrum interferences in the same group are detected and cancelled detected (from Table I users \#1, \#2, \#3, \#4). Code sequences that belong to different groups pass through the decoder without being detected (from Table I users \#5, \#6, \#7, \#8). As listed in Table I, the information of user\#1, which was coded as 011100 , has been modulated using ON-OFF Keying (OOK) technique as shown in Fig. 3. The optical pulses are then reflected to an FBG set, where specific wavelengths $\left(\lambda_{2} \lambda_{3} \lambda_{4}\right)$ are assigned to the chips of specific code given to the desired user. In the code sequences, the positions of the " $1 \mathrm{~s}$ " determine the center wavelengths of FBGs. The 
corresponding decoder decodes the received optical pulses. For the data to be recovered the decoder should have the same spectral response to the intended encoder [3]. The detected sequences comprise the HSAC code spectrum of the desired user in company with overlapping spectra from other interference of HSAC code sequences. The complementary decoder detects the complementary wavelengths, $\lambda_{1} \lambda_{5} \lambda_{6}$ of the intended user where the received wavelengths are processed via FBG sets. Subsequently the results are circulated to balanced photo-detectors [3]. A subtraction process is needed where a subtractor is used to strike the unwanted from the wanted signal. Finally, the original data is recovered after photo detections, low pass filter (LPF) and thresholding processes.

TABLE III. SPECTRAL BINS OF HSAC CODE WORDS FOR 8 USERS

\begin{tabular}{|c|c|c|c|c|c|c|c|c|c|c|c|c|}
\hline $\begin{array}{l}\text { Spectral } \\
\text { bin (nm) }\end{array}$ & $\begin{array}{c}\lambda .1 \\
1547.2\end{array}$ & $\begin{array}{c}\lambda .2 \\
1547.6\end{array}$ & $\begin{array}{c}\lambda, 3 \\
1548\end{array}$ & $\begin{array}{c}1.4 \\
1548.4\end{array}$ & $\begin{array}{c}\lambda .5 \\
1548.8\end{array}$ & $\begin{array}{c}\lambda .6 \\
1549.2\end{array}$ & $\begin{array}{c}\lambda .7 \\
1549.6\end{array}$ & $\begin{array}{c}\text { is } \\
1550\end{array}$ & $\begin{array}{c}1.9 \\
1550.4\end{array}$ & $\begin{array}{c}7.10 \\
1550.8\end{array}$ & $\begin{array}{c}\lambda .11 \\
1551.2\end{array}$ & $\begin{array}{c}\lambda 12 \\
1551.6\end{array}$ \\
\hline Users & & & & & & & & & & & & \\
\hline User\# 1 & & & & & & & & & & & & \\
\hline $\mathrm{C}_{(1,1)}$ & & & & & & & & & & & & \\
\hline User\# 2 & & & & & & & & & & & & \\
\hline $\mathrm{C}_{(1,2)}$ & & & & & & & & & & & & \\
\hline User\# 3 & & & & & & & & & & & & \\
\hline $\mathrm{C}_{(1,3)}$ & & & & & & & & & & & & \\
\hline User\# 4 & & & & & & & & & & & & \\
\hline $\mathrm{C}_{(1,4)}$ & & & & & & & & & & & & \\
\hline User\# 5 & & & & & & & & & & & & \\
\hline $\mathrm{C}_{(2,5)}$ & & & & & & & & & & & & \\
\hline User\# 6 & & & & & & & & & & & & \\
\hline $\mathrm{C}_{(2,6)}$ & & & & & & & & & & & & \\
\hline User\# 7 & & & & & & & & & & & & \\
\hline $\mathrm{C}_{(2,7)}$ & & & & & & & & & & & & \\
\hline User\# 8 & & & & & & & & & & & & \\
\hline $\mathrm{C}_{(2,8)}$ & & & & & & & & & & & & \\
\hline
\end{tabular}

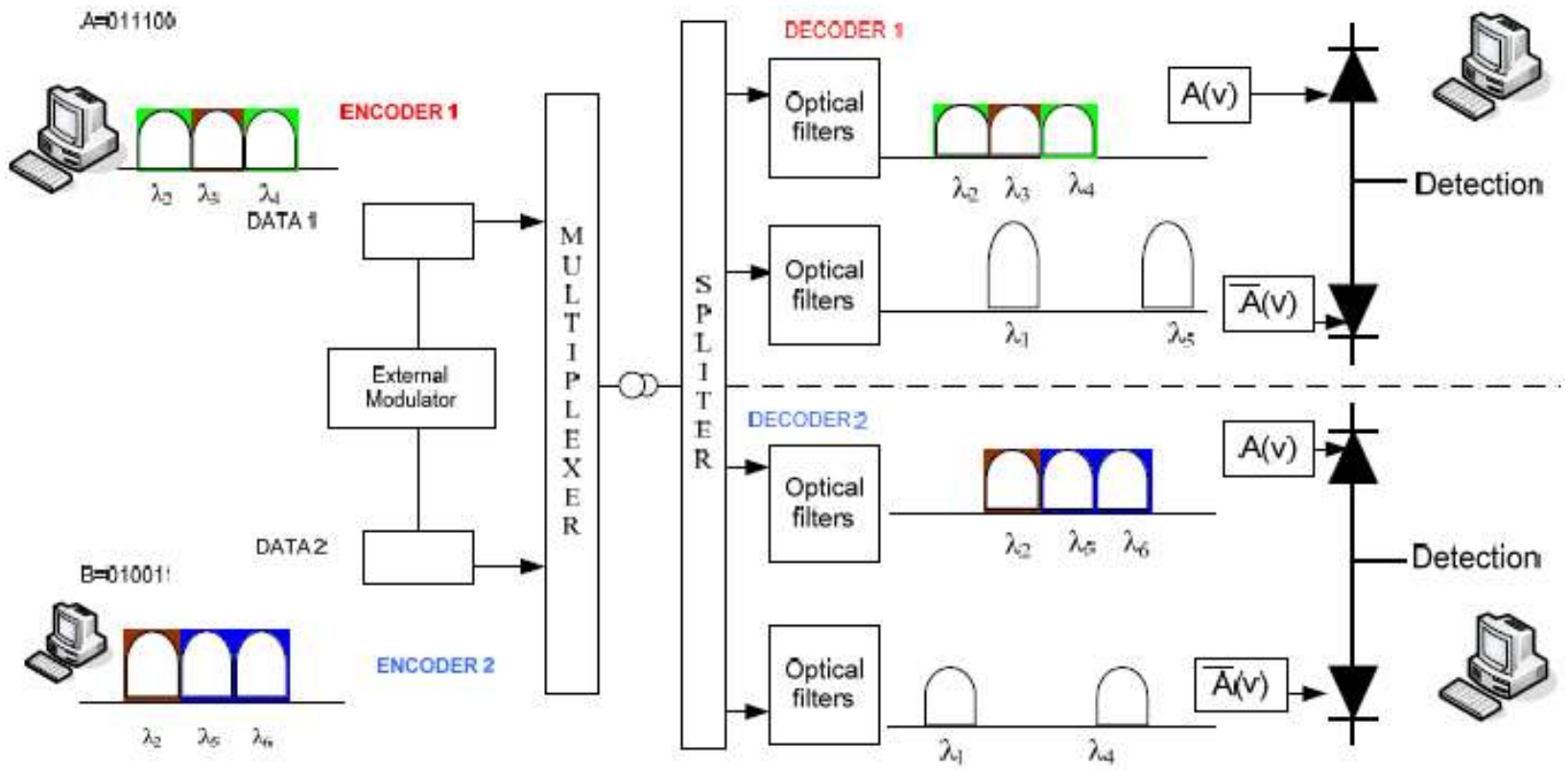

Fig. 3. Transmitter-receiver structure in LAN environment based on HSAC system 


\section{SYSTEM PERFORMANCE}

To analyze the system with transmitter and receiver as shown in Fig. 3 for HSAC, let $C_{x}(i)$ denotes the ith element of the $x$ th HSAC code sequences, the code properties based on XOR subtraction technique can be written as $[17,18]$ :

$\sum_{i=1}^{L} C_{x}(i) C_{y}(i)=\left\{\begin{array}{c}W, x=y \\ 1, x \neq y \\ 0, x \neq y\end{array}\right\} \quad \begin{gathered}\text { In the same group }(g=1) \\ \text { Not in the same group }(g \geq 2)\end{gathered}$

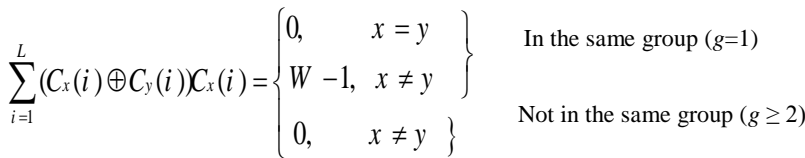

The condition of $x$ and $y$ in the same givup $(y-1)$ meamng that both code sequences are in $C S A C_{(1)}$ or $C S A C_{(2)}$ or $C S A C_{(\mathrm{m})}$ as shown Fig. 2. The condition of $x$ and $y$ not in the same group ( $g \geq 2$ ) meaning that one of the code sequence might be located in $C S A C_{(1)}$ and the other code sequence is located in $C S A C_{(2)}$ or $C S A C_{(\mathrm{m})}$. Therefore, the XOR operation of $\left(C_{x}(i)\right.$ $\left.\left.\oplus C_{y(i)}\right) C_{x}(i)\right)$ is valid for $x \neq y$ only. However, the cross correlation of $\left.\left(C_{x}(i) \oplus C_{y}(i)\right) C_{x}(i)\right)$ is valid for $x \neq y$ only in Eq. (15) while from Eq. (14), the cross correlation of $C_{x}(i) C_{y}(i)$ is $W$ when $x=y$.

Therefore, the MAI can be eliminated as the cross correlation $\sum_{i=1}^{L}\left(C_{x}(i) \oplus C_{y}(i)\right) C_{x}(i)$ can be subtracted from $\sum_{i=1}^{L} C_{x}(i) C_{y}(i)$ when $x \neq y$. Therefore, the decoder that computes Eq. (17) rejects the MAI coming from interfering users and obtains the desired information bits.

Thus

$$
\sum_{i=1}^{L} C_{x}(i) C_{y}(i)-\frac{\sum_{i=1}^{L}\left(C_{x}(i) \oplus C_{y}(i)\right) C_{x}(i)}{W-1}= \begin{cases}W, & x=y \\ 0, & x \neq y\end{cases}
$$

From Eq. (17) the weight is zero when $x \neq y$, means that an MAI impact can be completely removed by using XOR subtraction detection technique. Referring to the methods described in $[4,17]$ the SNRs of CSAC and HSAC can be calculated as follows.

$$
\begin{aligned}
S N R_{C S A C} & =\frac{\frac{\Re^{2} P_{s r}^{2}(W-1)^{2}}{L^{2}}}{\frac{P_{s r} e B \Re}{L}\left[(2(N-1)+W+1]+\frac{P_{s r}^{2} B \Re^{2} N}{2 \Delta V L^{2}}[W+1+2(N-1)]+\frac{4 K_{b} T_{n} B}{R_{L}}\right.} \\
S N R_{H S A C} & =\frac{\frac{\Re^{2} P_{s r}^{2}(W-1)^{2}}{L^{2}}}{\frac{P_{s r} e B \Re}{L}\left[(2(N-1)+W+1]+\frac{P_{s r}^{2} B \Re^{2} N}{2 \Delta V L^{2}}\left[W+1+\frac{2(N-1)}{g}\right]+\frac{4 K_{b} T_{n} B}{R_{L}}\right.}
\end{aligned}
$$

where $\mathfrak{R}$ is the photodiode responsivity, $P_{\mathrm{sr}}$ is the effective power of a broad-band source at the receiver, $e$ is the electron charge, $B$ is the electrical equivalent noise bandwidth of the receiver, $K B$ is Boltzmann's constant, $T_{\mathrm{n}}$ the absolute receiver noise temperature, $R_{\mathrm{L}}$ is the receiver load resistor, $\Delta V$ is the optical source bandwidth, $W, N, L$ are the code weight, the number of users and the code length respectively, as being the parameters of the HSAC code itself. The bit error rate (BER) is computed from the SNR using Gaussian approximation as [4-5].

The bit error rate (BER) can be calculated using

$$
P_{e}=\frac{1}{2} \operatorname{erfc}(\sqrt{S N R / 8})
$$

\section{RESULTS AND DISCUSSION FOR THEORETICAL PERFORMANCE ANALYSIS}

A sufficient amount of signal-to-noise ratio (SNR) is important because it states the quality of the signal in the system. BER and SNR are interconnected; a better SNR yields a better BER. In this section, the numerical results analyses are based on the SNR, BER, received output power for different type of noises, and noise power.

\section{A. Relationship between the number of active users (N) and $B E R$}

In Fig. 4, the BER is plotted against the number of active users when $P_{s r}=-10 \mathrm{dBm}$ at $622 \mathrm{Mbit} / \mathrm{s}$. From the figure, it is observed that the BER of HSAC code is lower compared to the CSAC, MQC and MFH codes even though the weight is far less, which is 4 in this case. For acceptable BER of $10^{-12}$ was achieved by the HSAC code with $\approx 140$ active users than for $\approx 65$ by CSAC code. This is evident from the fact that HSAC code has an in-phase cross correlation property that would eliminate the MAI effects by $g$ value. However, MQC and MFH codes used with a fixed in-phase cross-correlation exactly equal to one since the in-phase cross-correlation of these codes is always one, the PIIN induced in the system utilizing these codes is still significant, thus limiting the system performance for further improvement.

\section{B. The Effect of on system Performance by considering different Noises}

Fig. 5 shows the BER plotted against for HSAC code ( $\mathrm{W}=4, g=4)$ when the number of active users is 30 and the data rate is $622 \mathrm{Mbit} / \mathrm{s}$. The red dashed line with circles represents the BER of HSAC code performance when intensity noise, shot noise and thermal noise are considered. The blue line with squares represents the BER of CSAC code performance taking into account the effects of the intensity and thermal noises. The green solid line with stars represents the BER of CSAC code performance when intensity noise and shot noise are considered. It is shown that, when is large, both the shot and thermal noises are negligibly small compared to intensity noise, which becomes the main drawback factor of the system performance. 


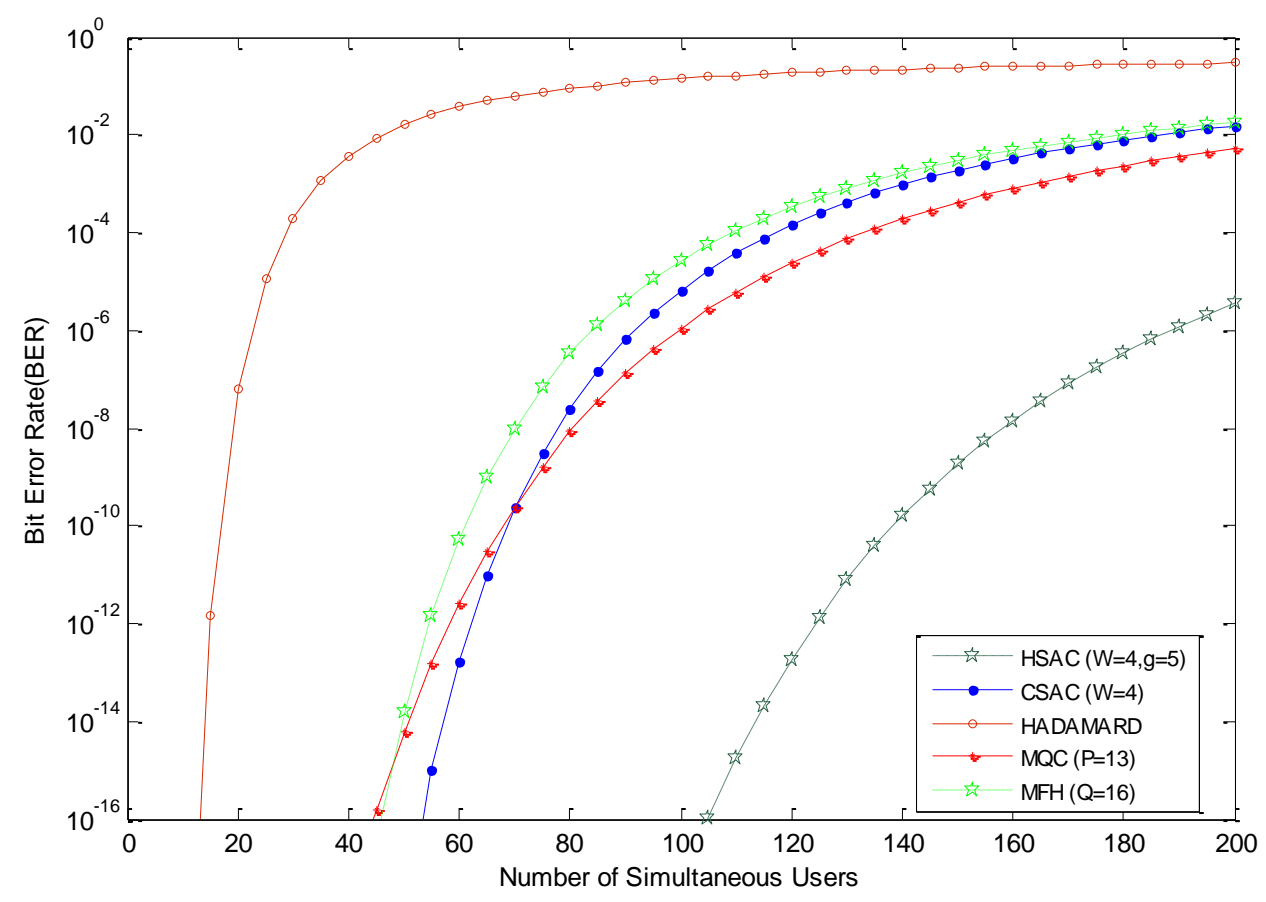

Fig. 4. BER versus number of active users when $P_{s r}=-10 \mathrm{dBm}$ at $622 \mathrm{Mb} / \mathrm{s}$

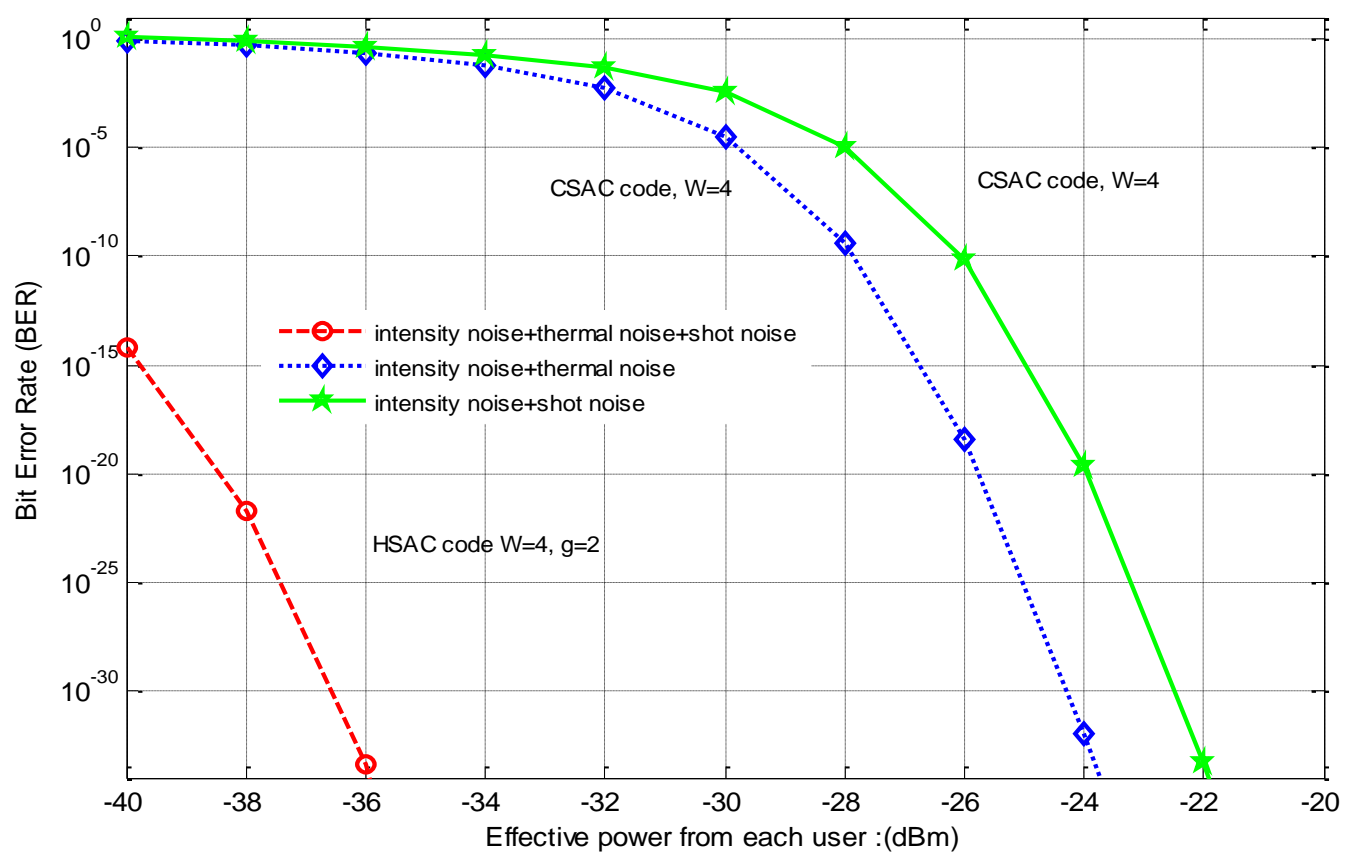

Fig. 5. BER versus effective source power $P_{s r}$ when the number of active users is 30 , taking into account the intensity noise, shot noise, and thermal noise at the data rate $622 \mathrm{Mb} / \mathrm{s}$ 


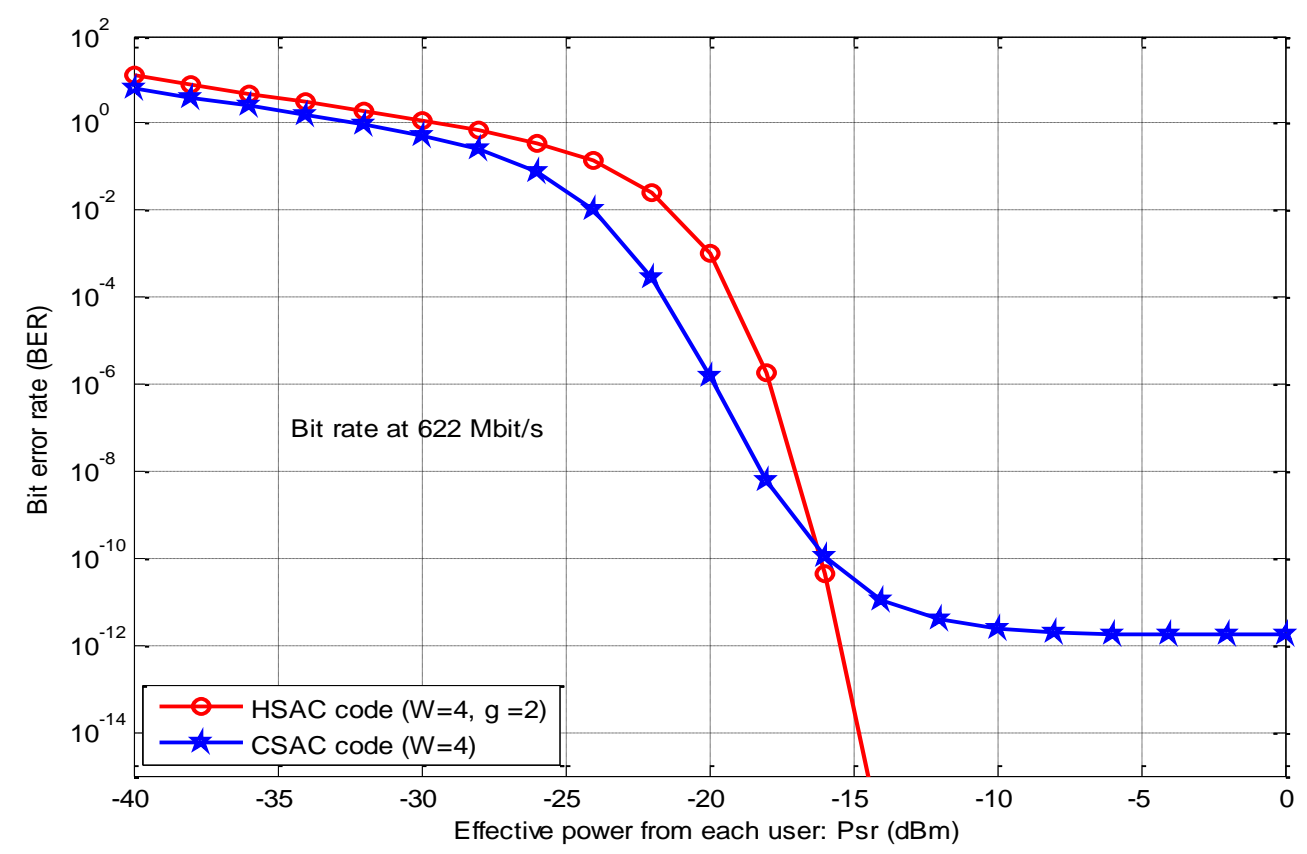

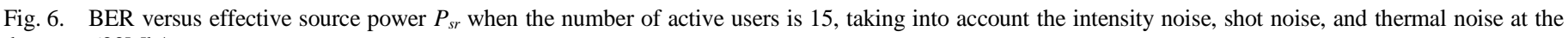
data rate $622 \mathrm{Mb} / \mathrm{s}$

On the other hand, when is low, the effect of intensity noise becomes minimal, and thus, the thermal source becomes the main factor that impairs the system performance.

Fig. 6 shows the BER variations with the effective power $P_{\text {sr }}$ when the number of active users is 15 at data rate of $622 \mathrm{Mb} / \mathrm{s}$ for each user, taking into account the effects of the intensity noise, thermal noise and shot noise for HSAC and CSAC codes.

CSAC is adopted with the parameters $W=4$ and $g=2$ (two groups); CSAC code is adopted with the parameter $W=4$. The figure shows that the effective power $P_{\mathrm{sr}}$ of acceptable BER of $10-10$ for the CSAC code is lower $\left(\approx P_{\mathrm{sr}}>-15 \mathrm{dBm}\right)$ than that for the CSAC code $\left(\approx P_{\mathrm{sr}}>0 \mathrm{dBm}\right)$ when the number of active users is the same. This is because the interference from other users is reduced by the value of $\mathrm{g}$ which is 2 in this case for HSAC while for CSAC is fixed as the number of simultaneous users increases. This figure reveals that HSAC code outperforms CSAC code by the magnitude of almost fifteen times.

For comparison purpose, the properties of the HSAC and MQC codes are listed in Table IV. Table IV shows that HSAC codes exist for any number of weight $\mathrm{W}$, free code size, and maximum in-phase cross correlation between any two code sequences is one. MQC code exists for prime number of users which limits the addressing flexibility of the system utilizing these codes to P2. HSAC has better performance and can support almost double number of users compared to MQC code.

\section{CONCLUSION}

In this paper, a new code family for SAC-OCDMA systems is presented. The properties of this code family have been proved and discussed. The results of system performance are compared with the reported codes. The HSAC code is a SAC code repeated in a diagonal fashion in wavelength domain. The in-phase cross correlation has maximum value of one $(\lambda=1)$ in the same group and zero $(\lambda=0)$ with codes in different groups.

It has been shown that the new code family can suppress intensity noise effectively and improve the system performance considerably. It shows that when a larger number of users are involved, the HSAC outperforms almost fifteen times the conventional ones and the bit-error rate is decreased as the number of groups $g$ is increased. An improvement in bandwidth utilization is reported where HSAC surpassed CSAC by double amount which promising burst environment like LAN to be its favorable candidate in high-speed applications. In addition to its improved performance, the HSAC codes are requiring less complexity in the encoder and decoder structure design.

\section{ACKNOWLEDGEMENT}

This project was supported by the Deanship of Scientific Research at Prince Sattam bin Abdulaziz University under the research project \# 2014/1/877. 
TABLE IV. COMPARISON BETWEEN HSAC AND MQC CODES

\begin{tabular}{|c|c|c|c|}
\hline Property & MQC-technique & HSAC-technique & Remarks \\
\hline$\lambda$ & 1 & $\leq 1$ & $\begin{array}{l}\text { 1- the maximum cross correlation is one between all code sets for } \\
\text { MQC } \\
\text { 2- the maximum cross correlation is zero when } g \geq 2 \text { for HSAC } \\
\text { codes }\end{array}$ \\
\hline Existence & Prime number & Any integer number & $\begin{array}{l}\text { More flexibility in code weight selection } \\
\text { for HSAC and limited selection for MQC codes }\end{array}$ \\
\hline Size & $\mathrm{P}^{2}$ & Free & Free cardinality for HSAC and limited codes \\
\hline Code length & $\mathrm{P}^{2}+\mathrm{P}$ & $L=g \frac{(W \times N)}{2}$ & \\
\hline $\begin{array}{c}\text { Number of variables and } \\
\text { parameters }\end{array}$ & $(\alpha, \beta, b, d$, and $k)$ & $W, \mathrm{~g}$ & $\begin{array}{l}\text { HSAC has two parameters in construction while MQC has five } \\
\qquad \text { parameters }\end{array}$ \\
\hline Matrix Form & Yes & Yes & less steps in HSAC compared to MQC \\
\hline
\end{tabular}

\section{REFERENCES}

[1] Frigo N, Iannone P, Reichmann K. Spectral slicing in WDM passive optical networks for local access, in IEEE Proc. Europ. Conf. Optical Commun. Tech. Dig, vol. 1, pp. 119-120, 1998.

[2] Froberg ., Henion, Rao H, Hazzard B, Parikh S, Romkey, Kuznetsov M, "The NGI ONRAMP test bed: Reconfigurable WDMtechnology for next generation regional access networks," J. LightwaveTechnol, vol.18, pp. 1697-1708, 2000

[3] Salehi J. A, Brackett C. A, "Code division multiple access techniques in optical fiber network-Part II: System performance analysis," IEEE Transaction on Communications, vol. 37, pp. 834-842, 1989.

[4] Zou Wei, Shalaby H. M. H, Ghafouri-Shiraz H, "Modified Quadratic Congruence codes for Fiber Bragg-Grating-Based SAC-OCDMA," Journal of Lightwave Technology, vol. 19, pp. 1274-1281, 2001.

[5] Wei Z, Ghafouri-Shiraz H, "Code for spectral amplitude-coding optical CDMA systems," J. Lightwave Technol, vol. 20, pp. 1284-1291, 2002.

[6] Abd T. H, Aljunid S. A, Fadhil H. A, Ahmad R. B, Junita M. N, "Enhancement of performance of a hybrid SAC-OCDMA system using dynamic cyclic shift code," Ukr. J. Phys. Opt, vol. 13, pp. 12-27, 2012.

[7] Park E, Mendez A.J, Garmire E. M, "Temporal/spatial optical CDMA networks-design, demonstration, and comparison with temporal networks," IEEE Photonics Technol Lett, vol. 4, pp. 1160-1162, 1992.

[8] Yim R.M.H, Chen L.R, Bajcsy J, "Design and performance of 2-D codes for wavelength-time optical CDMA," IEEE Photonics Technol. Lett, vol. 14, pp. 714-716, 2002.

[9] Mendez Antonio. J, Gagliardi Robert M, Vincent J. Hernandez, Bennett Corey V, Lennon William J, "Design and performance analysis of wavelength/time (W/T) matrix codes for optical CDMA," IEEE J. Lightwave Technol, vol. 21, pp. 2524-2533, 2003.
[10] Song-Ming Lin, Jen-Fa Huang, Chao-Chin Yang, "Optical CDMA network codecs with merged-M-coded wavelength-hopping and primecoded time-spreading," Opt. Fiber Technol, vol. 13, pp. 117-128, 2007.

[11] Sheng Peng Wan, Hu Yu, "Two-dimensional optical CDMA differential system with prime/OOC codes," IEEE Photonics Technol. Lett, vol. 13, pp.1373-1375, 2001.

[12] Yim R.M.H, Bajcsy J, Chen L.R, "A new family of 2-D wavelengthtime codes for optical CDMA with differential detection," IEEE Photonics Technol. Lett, vol. 15, pp.165-167, 2003.

[13] Heo H, Seong-sik Min, Won Yong Hyub, Yeon Y, Bong Kyu Kim, Byoung Whi Kim, "A new family of 2-D wavelength-time spreading code for optical code-division multiple-access system with balanced detection," IEEE Photonics Technol. Lett, vol. 16, pp. 2189-2191, 2004.

[14] Yang C. C, Huang J. F, "Two-Dimensional M-matrices coding in spatial/frequency optical CDMA networks," IEEE Photon. Technol. Lett, vol.15, pp. 168-170, 2003.

[15] Smith E. D. J, Blaikie R. J, Taylor D. P, "Performance enhancement of spectral-amplitude-coding optical CDMA using pulse-position modulation," IEEE Trans. Commun, vol. 46, pp. 1176-1185, 1998.

[16] Chao-Chin Yang, "Hybrid wavelength-division multiplexing/spectralamplitude-coding optical CDMA system," IEEE Photon. Technol. Lett, vol. 17, pp. 1343-1345, 2005.

[17] Hassan Yousif Ahmed, Nisar K.S, "Diagonal Eigenvalue Unity (DEU) code for spectral amplitude coding-optical code division multiple access," Optical Fiber Technology, vol. 19, pp. 335-347, 2013.

[18] Hassan Yousif Ahmed, Nisar K.S, "Reduction of the fiber dispersion effects on MAI for long span high-speed OCDMA networks using Diagonal Eigenvalue Unity (DEU) code," Optik, vol. 124, pp. 57655773, 2013.

[19] Goodman J.W, Statistical optics. Wiley: New York, 1985. 